\title{
Gradhiva
}

GRADHIV

Revue d'anthropologie et d'histoire des arts

Voir et reconnaître. L'objet du malentendu

\section{Les « Chroniques éthiopiennes » de Marcel Griaule} L'ethnologie, la littérature et le document en 1934

The "Ethiopian chronicles" by Marcel Griaule. The ethnology, the literature and the document in 1934

\section{Vincent Debaene}

\section{OpenEdition}

Journals

Édition électronique

URL : http://journals.openedition.org/gradhiva/955

DOI : $10.4000 /$ gradhiva.955

ISSN : $1760-849 x$

\section{Éditeur}

Musée du quai Branly Jacques Chirac

\section{Édition imprimée}

Date de publication : 15 novembre 2007

Pagination : 86-103

ISBN : 978-2-915133-55-4

ISSN : 0764-8928

\section{Référence électronique}

Vincent Debaene, «Les « Chroniques éthiopiennes » de Marcel Griaule », Gradhiva [En ligne], 6 | 2007, mis en ligne le 15 novembre 2010, consulté le 04 mai 2019. URL : http://journals.openedition.org/ gradhiva/955; DOl : 10.4000/gradhiva.955

Ce document a été généré automatiquement le 4 mai 2019.

(C) musée du quai Branly 


\title{
Les «Chroniques éthiopiennes » de Marcel Griaule
}

\author{
L'ethnologie, la littérature et le document en 1934 \\ The "Ethiopian chronicles" by Marcel Griaule. The ethnology, the literature and \\ the document in 1934
}

Vincent Debaene

« Mais moi, jeune Français, né au Nord de Paris, suis-je bien sûr de deviner ce qu'éprouvaient ces âmes italiennes de l'an 1559 ? Je puis tout au plus espérer de deviner ce qui peut paraître élégant et piquant aux lecteurs français de 1838. » Stendhal, « La duchesse de Palliano » (1838), Chroniques italiennes.

1 De la « construction du savoir » à la « fabrique du texte », en passant par la " production des faits scientifiques ", de nombreuses formules semblent témoigner, depuis quelques années déjà, d'une nouvelle lucidité à l'endroit des discours savants, qui toutes semblent dire: «nous ne sommes plus dupes" (de l'apparente neutralité du savant, de la naturalisation des faits sociologiques, de la fausse transparence du récit, etc.). L'ethnologie, science si visiblement « impure » (de l'ailleurs à l'ici, du terrain à la note, de l'humain au texte), se prête tout particulièrement à ce type d'approche. Au cours des vingt dernières années, les discours anthropologiques des "pères » de la discipline ont fait l'objet de nombreuses déconstructions génétiques à partir des correspondances, des archives et des différents états du texte antérieurs à la publication. Les écrits de Marcel Griaule ne font pas exception. Éric Jolly et Jacques Mercier ont ainsi montré que les «notes ethnographiques » et même les «monographies» des années trente étaient, le plus souvent, le fruit d'un collage de fiches, fiches elles-mêmes rédigées sur le terrain conformément à un protocole défini très tôt et qui n'a pour ainsi dire pas varié au long de la carrière d'« africaniste » de l'auteur'. 
On se propose ici de procéder différemment, de façon non plus génétique ou "verticale ", mais «horizontale» en quelque sorte, en opérant une coupe transversale et en considérant tous les écrits publiés au retour du terrain, en s'intéressant donc moins à la construction du savoir qu'à sa distribution selon les lieux et les publics.

3 L'ethnologie française de l'entre-deux-guerres est en effet caractérisée par un étonnant éclatement bibliographique. Autour d'une seule expédition se développe toute une constellation de textes, très divers dans leur forme et leur visée : monographie pour la collection des Travaux et mémoires de l'Institut d'ethnologie, articles dans des quotidiens (Paris-Soir), récit de l'expédition dans une revue «intellectuelle» (Revue de Paris, NRF), publication de documents dans des revues d'art (Arts et métiers graphiques, Documents), rapports d'expédition dans le Journal de la Société des africanistes ou américanistes, guide d'exposition pour le « Troca » ou le musée de l'Homme, et récit de voyage chez Grasset ou Gallimard. La première expédition menée par Griaule est un cas exemplaire, car elle donne lieu à quantités d'articles de différents ordres, ainsi qu'à un long récit, Les Flambeurs d'hommes, publié en 1934. Ce curieux ouvrage peut être lu comme un révélateur des contradictions de l'ethnologie de cette époque, et il témoigne en particulier du rapport coupable de cette discipline à la littérature.

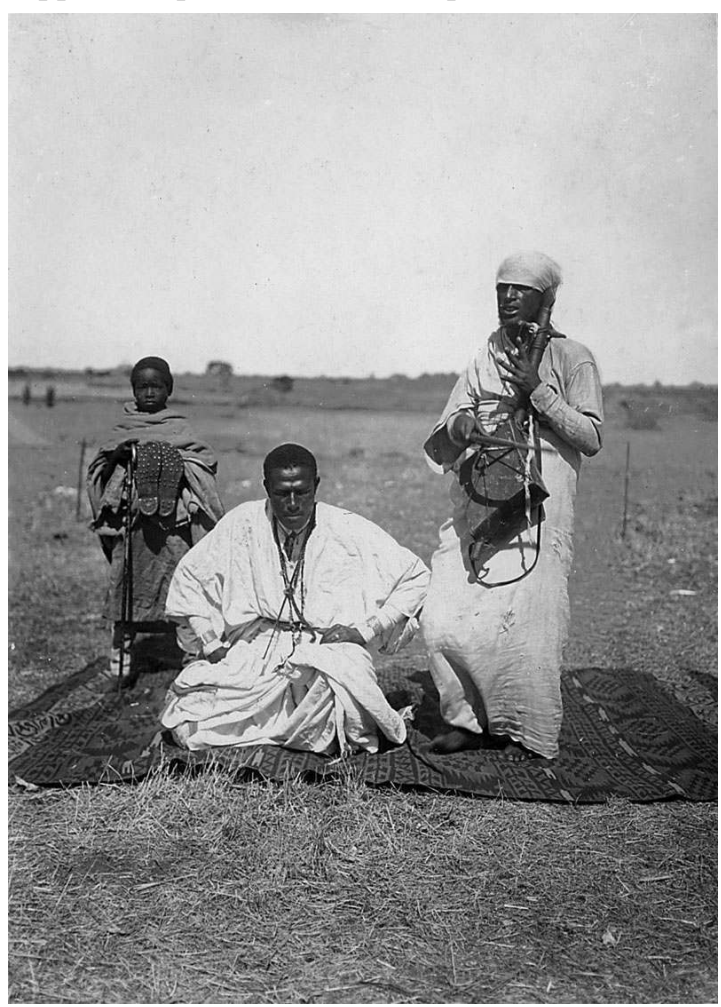

Fig. 1 Musiciens du ras Haïlou.

(c) musée du quai Branly, photo Marcel Griaule.

\section{Les Flambeurs d'hommes et la première « mission Griaule »}

4 La «mission d'études ethnographiques et linguistiques » menée par Marcel Griaule en Éthiopie - qui, rétrospectivement, apparaîtra comme la première « mission Griaule » quitte Paris le 25 septembre 1928; elle est mandatée par le ministère de l'Instruction 
publique et des Beaux-Arts, et soutenue par l'Académie des inscriptions et belles-lettres, l'Institut d'ethnologie de l'Université de Paris, et la Fondation nationale pour l'étude des sciences et des civilisations étrangères ${ }^{2}$. Avec son compagnon, Marcel Larget, Griaule s'établit en janvier 1929 auprès du ras Haïlou au camp d'Addiet (région du Godjam) où il séjourne cinq mois, préoccupé essentiellement d'ethnographie religieuse avec pour «but principal [...] l'étude de la civilisation classique éthiopienne » (Griaule $1930: 453)$. Griaule prétend aller à l'encontre du préjugé habituel qui conduit l'ethnographie à délaisser les populations chrétiennes (les Amhara sont chrétiens depuis le Ive siècle). Les textes publiés au retour peuvent être distribués en deux ensembles : d'une part, une série de travaux savants, constituée pour l'essentiel des deux volumes Silhouettes et graffiti abyssins et Jeux et divertissements abyssins (1933 et 1935); d'autre part, Les Flambeurs d'hommes, ouvrage présenté par Griaule comme « une description objective de certaines péripéties de [son] voyage en Abyssinie », publié chez Calmann-Lévy en 1934.

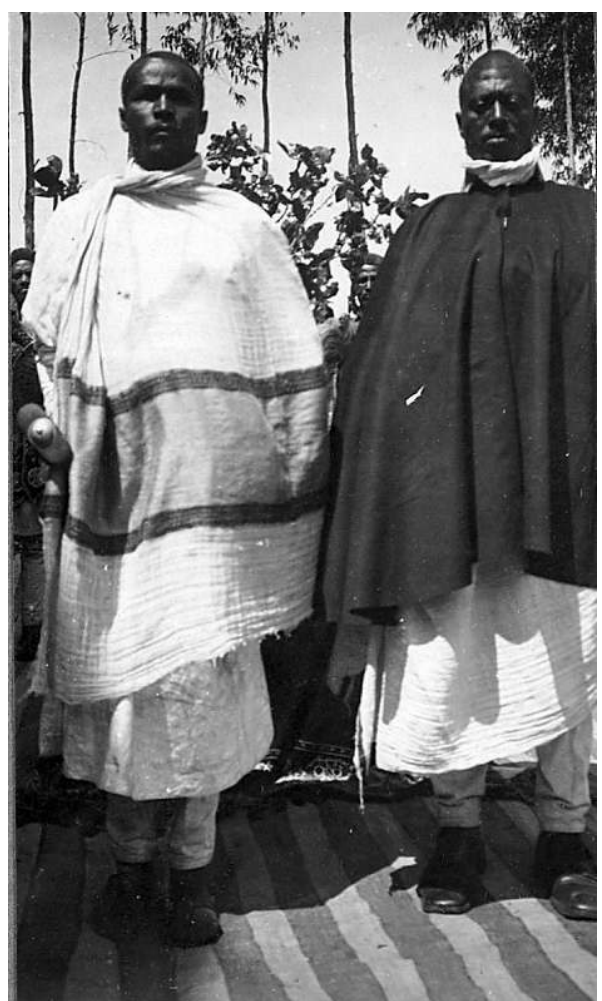

Fig. 2 Notables du Godjam.

(c) musée du quai Branly, photo Marcel Griaule.

Les Flambeurs d'hommes est un livre très singulier, en raison d'abord de sa relative opacité. Il n'est pas mal écrit, mais bizarrement sentencieux, et comporte de brutales ruptures de ton et de registre. On n'y trouve aucune précision quant aux dates, aux membres, à l'activité ou aux projets de la mission. Seul un lecteur attentif pourra comprendre, par exemple, que le "personnel» européen de la mission se réduit en réalité à deux personnes : Griaule lui-même et Marcel Larget, qualifié de « chimiste » dans Silhouettes et graffiti abyssins, homme de terrain plus que savant, et qui participera également à la mission Dakar-Djibouti. Très elliptique, ne s'engageant que rarement dans une description et jamais dans une explication, ne faisant que brièvement mention du travail d'enquête, Les Flambeurs d'hommes semble généralement manifester une sorte de méfiance envers toute perspective " communicationnelle », et il n'est pas toujours facile de saisir 
tout simplement de quoi il est question. Les seules mentions référentielles sont celles des toponymes, mais ces derniers, littéralement traduits (« Abbaye-de-Marc », « Zaguié-Borddu-Lac », "Repos-de-Marie », etc.), au lieu d'ancrer le récit dans la réalité, le place d'emblée au plan du mythe. C'est en définitive une sorte d'Éthiopie archaïque et rêvée qui est mise en scène, une société où même l'esclave et le paysan sont des maîtres de langue et où les hommes, jusqu'aux plus humbles, font montre d'une grande noblesse et d'un sens de l'honneur sans équivalents en Europe. L'ouvrage s'ouvre sur ces mots :

«Le Nil est cette chose qui empêche le caravanier de dormir. Passer un fleuve, surtout s'il roule dans des bas-fonds, c'est se faufiler entre les doigts du diable.» (FH : 31)

Bien qu'il propose une vision manifestement idéalisée de l'Éthiopie (comme l'atteste, du reste, la désignation même du pays qui n'apparaît que sous le nom d'Abyssinie), Griaule justifie très curieusement le livre par un souci de vulgarisation :

« Je me hâte d'ajouter que les documents recueillis ont donné lieu à de nombreuses publications scientifiques et que le présent ouvrage a été composé dans un tout autre esprit. [...] les érudits consentent rarement [...] à écrire pour le grand public [...]. Mais en ce qui concerne l'ethnographie, cette science vivante entre toutes, et d'autre part étant donné que le public subventionne directement les missions officielles, il m'a semblé possible, voire nécessaire, en tout cas aimable, de descendre les pentes orgueilleuses de l'érudition et de présenter à un plus grand nombre de lecteurs un ouvrage lisible. Il ne s'agit pas là d'un journal de route ni d'un récit romancé, mais d'une description objective de certaines péripéties de mon premier voyage en Abyssinie. » $(\mathrm{FH}: 27)$

7 Les Flambeurs d'hommes connaît un succès certain lors de sa parution; il compte 43 éditions en 1936 et est déjà, à cette date, traduit en anglais, en allemand et en tchèque. En 1935, il obtient le prix Gringoire, grâce en particulier à l'activisme de Paul Rivet et Georges-Henri Rivière auprès du jury (où siègent, entre autres, André Maurois, Francis Carco, Jacques de Lacretelle et Joseph Kessel). On peut, sans grand risque d'erreur, attribuer une bonne part de ce retentissement à l'épisode qui donne son titre au livre: le supplice de la « mort par la mousseline », dont le récit est paru en «bonnes feuilles » dans la Revue de Paris dès avril 1934. Dans le cinquième chapitre, Griaule relate le procès, puis le supplice et l'exécution d'un homme qui aurait tenté d'assassiner le rasHaïlou ; le coupable est emmailloté dans des bandes de mousseline trempées dans la cire chaude, puis brûlé vif en public :

«Vision d'enfer, cette expression n'est pas trop forte; on conviendra que dans une pareille exaltation il suffit qu'une flamme émise par un homme vivant ait cinq mètres de hauteur pour paraître infernale. » (FH : 126) 


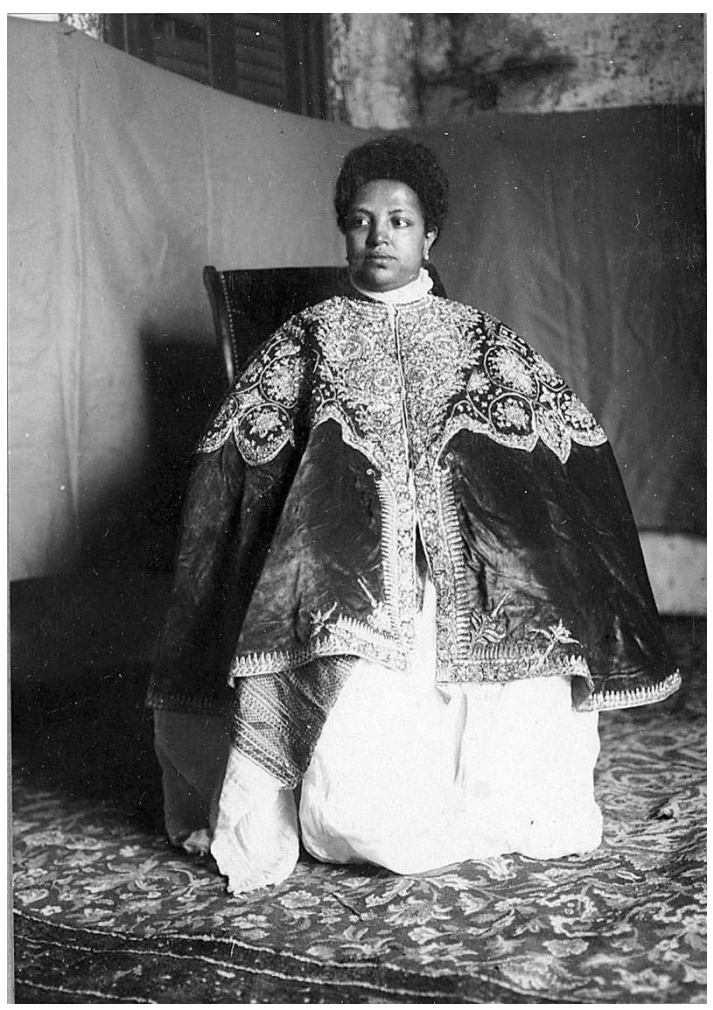

Fig. 3 La fille du ras Haïlou.

(c) musée du quai Branly, photo Marcel Griaule.

Trois caractéristiques principales font l'originalité du livre dans l'ensemble des récits ethnographiques de l'époque. On y trouve d'abord un certain nombre d'affabulations, à commencer par le supplice de la «mort par la mousseline » lui-même qui est une pure et simple invention ${ }^{3}$. L'affabulation est très fréquente dans les récits de voyage de l'entredeux-guerres (il n'est pas un «explorateur " qui, à son retour en France, ne prétende avoir assisté à des scènes d'anthropophagie ou écouté le chant des tambours en peau humaine). Mais si les récits ethnographiques comportent un certain nombre d'erreurs, d'approximations, de déformations, aucun d'entre eux ne propose de fiction aussi éhontée. Ensuite, Les Flambeurs d'hommes ne compte aucune photographie. C'est un cas unique ; l'insertion de photographies est systématique dans les récits ethnographiques de la période, et plus largement dans les récits de voyage en terre exotique ${ }^{4}$. Enfin et surtout - curiosité absolument sans équivalent dans la production de l'époque -, Les Flambeurs d'hommes est écrit à la troisième personne. Griaule y apparaît comme «le Blanc », «le Franc » ou «l'Européen ». Ainsi peut-on lire, par exemple, à propos de la descente dans les « bas-fonds » de la vallée du Nil :

« Il y avait eu, la veille, une longue mise au point de la descente entre l'Européen et les caravaniers. La discussion aurait pu se terminer mal s'il y avait eu des pistolets à proximité. La raison en était cette inexplicable impatience de l'étranger à vouloir s'enfoncer dans des régions diaboliques, sans écouter les doléances des caravaniers qui continuaient irrespectueusement à tremper leurs doigts dans un pot de ragoût ; il s'était écrié, à bout d'arguments :

- Vous m'avez promis le passage pour mercredi. Si nous ne passons pas mercredi, nous serons retardés de trois jours, puisqu'il n'est pas permis de descendre le jeudi et le vendredi.

- Quand on peut retarder un malheur de trois jours, avait dit un homme de poids, on remercie Dieu et on s'assoit ! 
Sur quoi il y avait eu des coups donnés par le Blanc et non rendus, car un Blanc est toujours homme de gouvernement, qui fait éclater des complications dès qu'on le touche. » (FH : 35-37)

On essaiera donc ici de rendre raison des bizarreries de ce livre, en considérant non pas la psychologie de Griaule, mais plutôt cet éclatement des écrits publiés au retour, et la place singulière qu'y occupe Les Flambeurs d'hommes. L'hypothèse générale est la suivante : la distribution en deux ensembles - les deux «monographies ", d'un côté, et Les Flambeurs d'hommes, de l'autre - est la conséquence d'une contradiction fondamentale entre, d'une part, la dynamique institutionnelle de fondation de l'ethnologie (universitaire) et, d'autre part, la théorie du fait social comme fait moral. D'un côté, l'ethnologie comme discipline s'élabore autour du musée; il faut rassembler les faits, collecter les objets et les sauvegarder avant qu'il ne soit trop tard. Il s'agit d'un modèle documentaire, inspiré de l'archéologie et de l'histoire; le travail savant est d'abord un travail d'établissement du fait: il faut remplir les vides, combler les lacunes du fichier des "archives de l'humanité ». De l'autre, cette dynamique institutionnelle (et l'épistémologie un peu lâche qui la sous-tend) se heurte à l'idée que le fait ethnographique n'est pas réductible au document, que le document dans sa matérialité ne suffit pas à l'établir. Le fait social est un fait d'ordre moral ; il détermine des façons de penser, des façons de sentir. Ce sont des "états de conscience", selon une expression de Marcel Mauss, et il est bien difficile d'inférer de tels états de conscience à partir du masque ou du mythe (voir Debaene 2006). De là, la nécessité d'un second livre, une sorte de "supplément au voyage de l'ethnographe ", chargé de compenser les insuffisances du document. C'est cette fonction que tâche de remplir Les Flambeurs d'hommes.

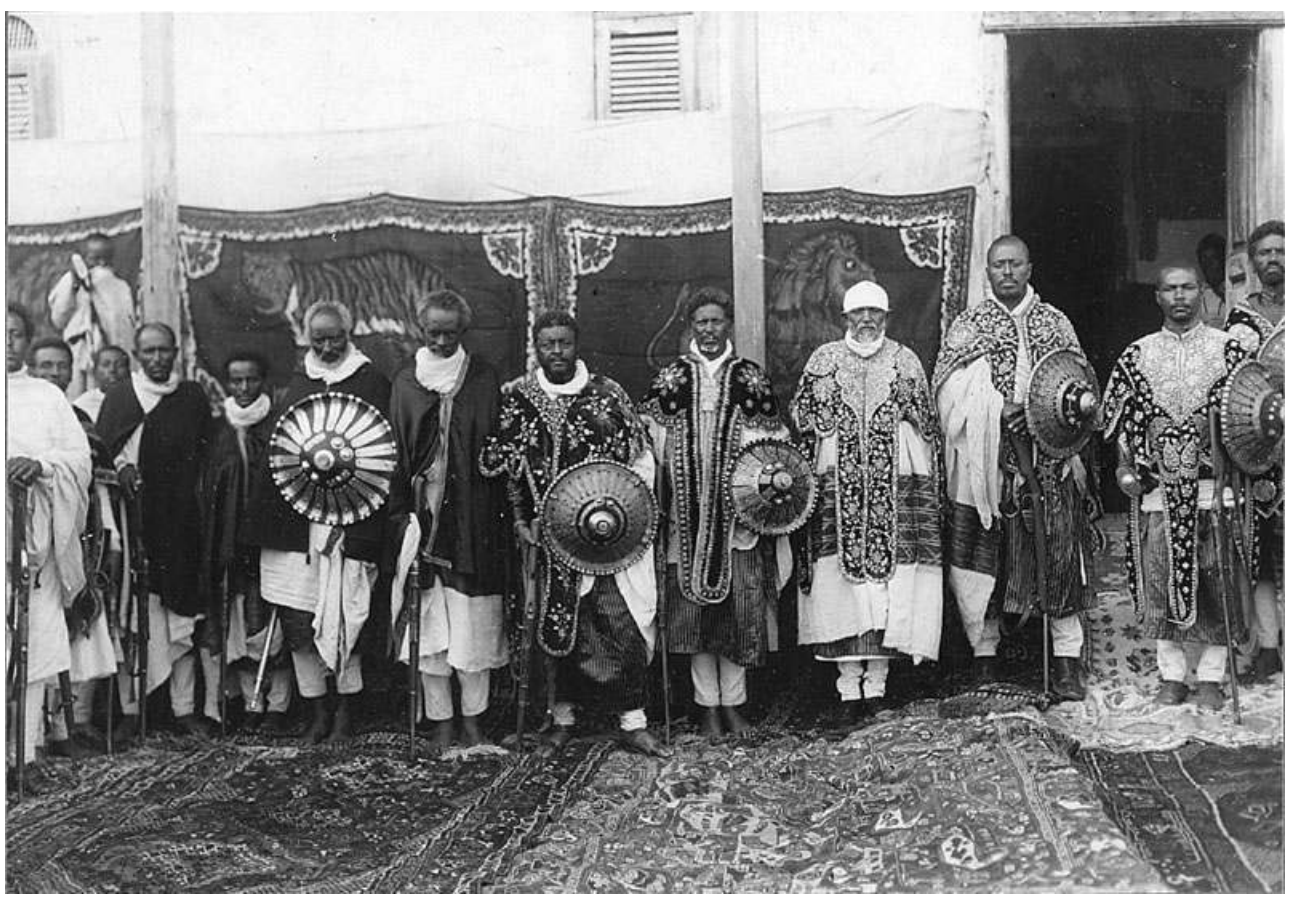

Fig. 4 Les grands chefs du ras Haïlou.

(c) musée du quai Branly, photo Marcel Griaule. 


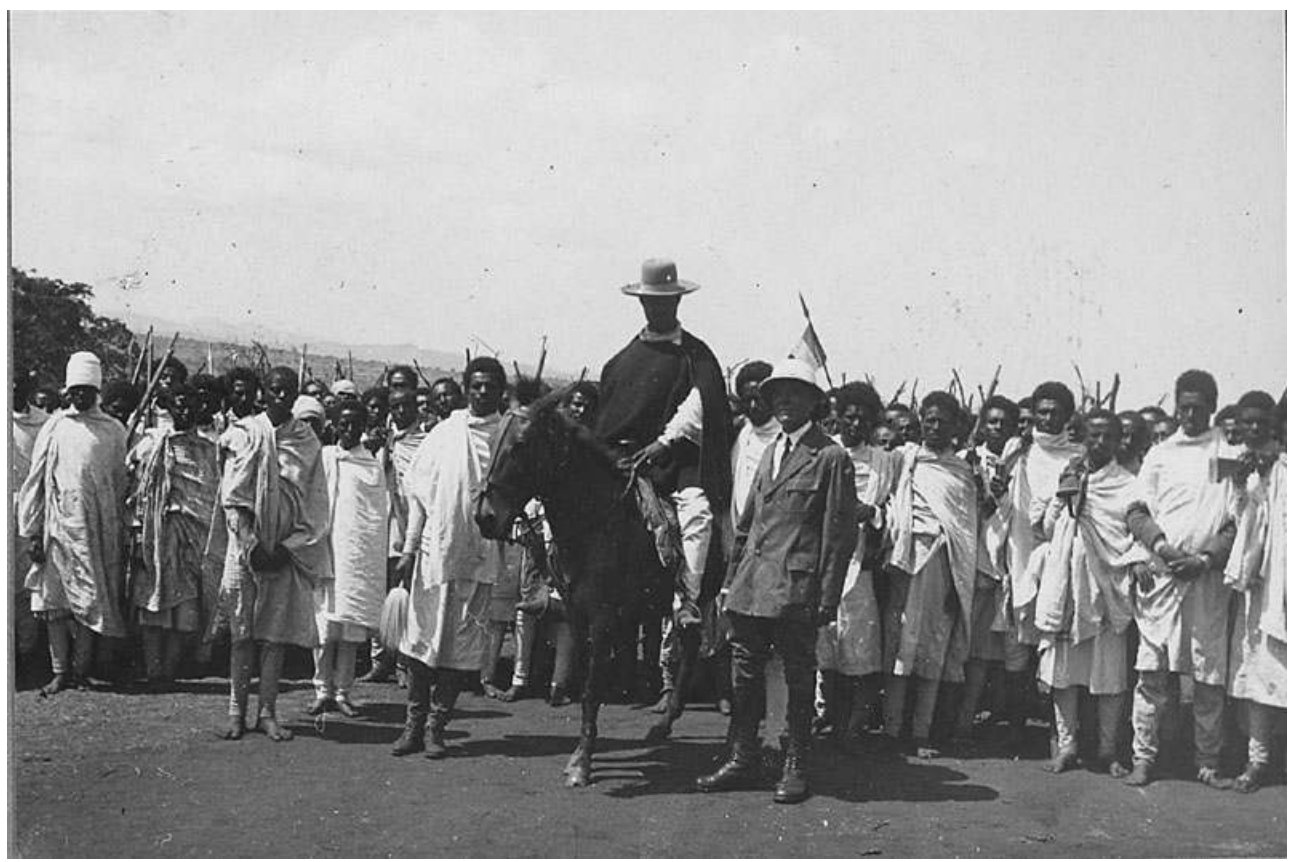

Fig. 5 Le ras Haïlou aux abords de sa résidence.

(c) musée du quai Branly, photo Marcel Griaule.

\section{La rhétorique, le document, l'atmosphère}

Pour un lecteur d'aujourd'hui, le travail scientifique de Griaule est quelque peu surprenant ; il paraît marqué par une sorte de délire d'exhaustivité, un désir d'inventaire jusqu'au vertige. Tout doit être archivé et recensé : la forme des faites de toit, les différents tours à travailler la corne en Abyssinie septentrionale, les nœuds et jeux de ficelles au Godjam, et, bien sûr, les mythes, les croyances, les légendes... Ce n'est pas tant cette volonté de recensement qui est étonnante que le refus obstiné de l'interprétation qui l'accompagne, ainsi que l'absence de questionnement quant aux principes de la collecte. On peut considérer par exemple Jeux et divertissements abyssins. L'ouvrage de 1935 comporte 260 pages et 25 planches ; on y trouve plusieurs centaines de fiches classées par thèmes et organisées par un questionnement : quoi/ quand/qui/où.

« Lance

Des tiges de sorgho sont tressées en forme de fer de lance qu'on emmanche d'une hampe. Les tiges sont teintes, après le tressage, avec de la sève de sycomore.

Février à avril

Garçons Tigré

[chapitre « Jouets », p. 7]

Poupée

On confectionne de grossières poupées avec des chiffons.

Toute l'année

Filles Choa

[chapitre « Jouets », p. 13]

Bruits

Les deux joues sont violemment frappées avec les deux poings.

Toute l'année

Garçons Wollo

La main est placée sous l'aisselle opposée, la paume ménageant une poche d'air. Le bras est vivement ramené le long du corps en restant replié. On provoque ainsi un 
pet sonore.

Toute l'année

Enfants et jeunes gens Wollo, Tigré

[chapitre « Musique », p. 10]»

11 Dans l'introduction, Griaule explique ce parti pris de littéralité :

«Il ne m'a pas paru indispensable d'établir un classement méthodique des documents recueillis. Le danger de systématiser à faux eût été inévitable, étant donné le caractère composite d'un grand nombre de jeux, surtout si on les envisage dans la totalité de leurs variantes [...]. Je considère hors de mon sujet de dégager le caractère des jeux abyssins. J'estime que l'ethnographe, même après la plus ample moisson, peut s'abstenir de systématiser, car il n'est pas de moisson si abondante qui ne puisse être multipliée dans des proportions inattendues quand on fouille à nouveau le terrain de recherches. Il est d'ailleurs juste temps que des ethnographes se consacrent entièrement à l'observation pure et simple des faits et laissent à d'autres spécialistes le soin d'utiliser leurs documents [...]. » (Griaule 1935 : 4-6)

Le travail ethnographique est donc un travail de collecte, et surtout pas un travail théorique. À cela, il y a deux raisons. D'une part, il y a urgence : les sociétés primitives sont en passe de disparaître ; des milliers de coutumes, de langues, de mythes ont déjà été engloutis, et il faut se hâter de sauver ce qui peut encore l'être. D'autre part, la découverte d'un seul fait, demain, pourrait tout remettre en cause. C'est un lieu commun de la période, et propagé avec vigueur par Griaule dans tous ses écrits : il est trop tôt pour l'anthropologie. Implicitement, la perspective est inductive: il s'agit de réunir un maximum de faits et c'est seulement quand le fichier sera complet que l'on pourra élaborer des lois. L'ethnologie est donc définie par Griaule comme une archéologie par anticipation, à deux titres: d'abord, parce qu'elle s'inscrit dans une perspective de sauvegarde; ensuite, et surtout, parce qu'à la manière dont la découverte d'un tesson de poterie peut démentir toute une théorie ou réduire à néant une hypothèse de datation, un fait ethnographique est susceptible de remettre en cause toute construction 
anthropologique. Inutile, donc, de s'aventurer dans des élaborations théoriques ; la seule responsabilité qui incombe au savant est celle de l'établissement du fait.

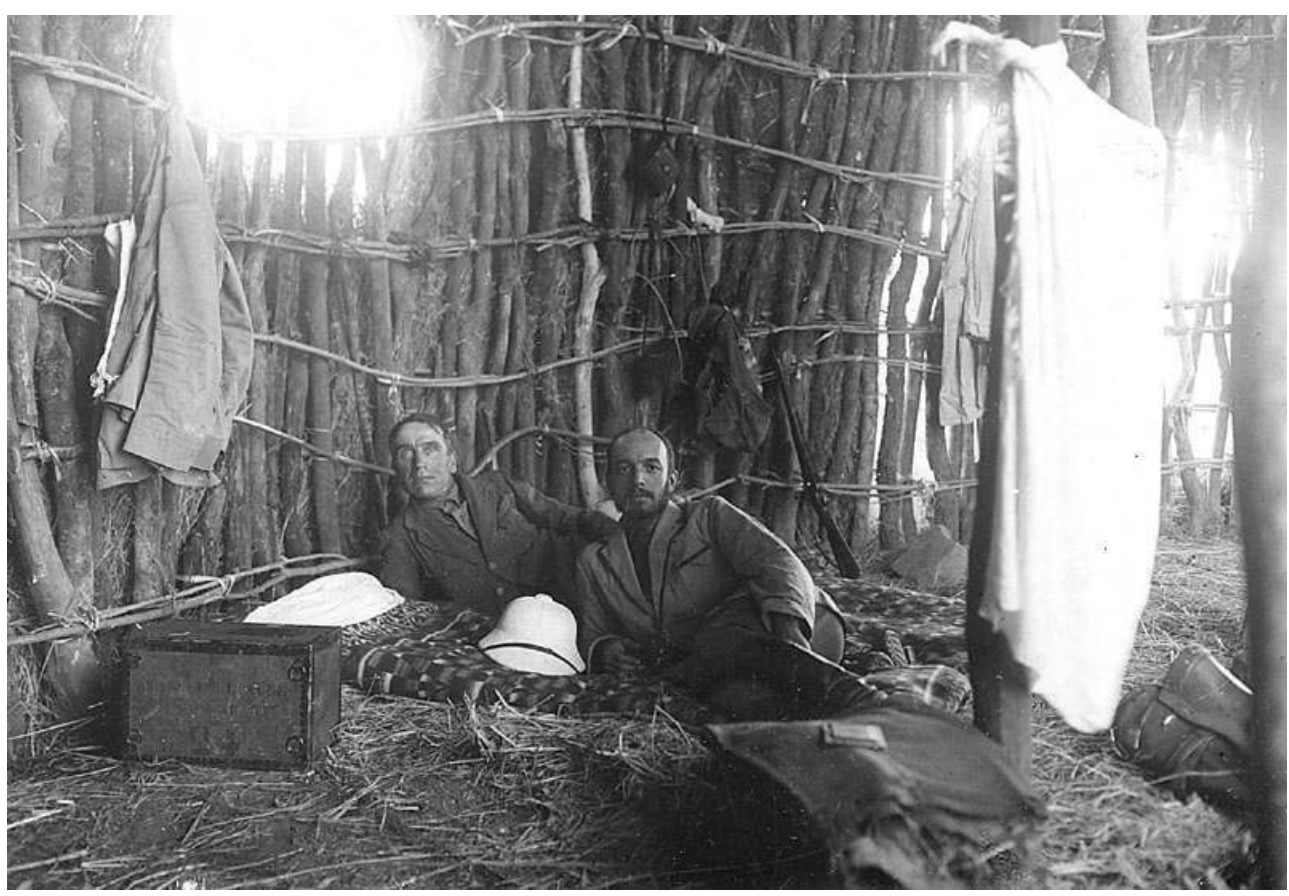

Fig. 6 Marcel Griaule et Marcel Larget lors de la mission Griaule en Éthiopie (1928).

(c) musée du quai Branly, photo Marcel Griaule.

Le modèle implicite qui préside à cette définition de l'ethnologie est celui de l'histoire méthodique ("positiviste») de la fin du XIX siècle: le travail savant consiste en une accumulation de documents, doublée d'un travail critique sur les sources, mais dès lors que le document est établi, l'historien n'a plus qu'à l'enregistrer passivement - ce que l'histoire des Annales dénoncera plus tard comme le « culte du fait » ou le « fétichisme du document». L'histoire méthodique s'est elle-même d'abord définie contre la « littérature ", c'est-à-dire contre la rhétorique : l'histoire ancienne, celle de Michelet ou celle de Guizot, était trop pittoresque, trop séduisante. Ces historiens étaient avant tout des littérateurs, ils gagnaient la conviction du lecteur en raison de leur style brillant, mais ne se fondaient pas sur des faits rigoureusement établis. Dans l'introduction qui ouvre le premier numéro de la Revue historique, Gabriel Monod compare ainsi les "progrès des sciences historiques» en Allemagne et en France, et fait l'éloge de la recherche allemande, injustement taxée d'érudition stérile :

«Les idées générales y abondent au contraire, seulement ce ne sont pas des fantaisies littéraires, inventées en un moment au caprice et pour le charme de l'imagination: ce ne sont pas des systèmes et des théories destinées à plaire par leur belle apparence et leur structure artistique. » (Monod 1876 : 29)

Plus loin, il déplore que le "génie de la nation» française soit trop «enclin aux séductions de l'imagination et de l'art ", et regrette que même les plus érudits parmi les historiens français soient "des littérateurs avant d'être des savants »: "Ce qui leur importe dans leurs écrits, c'est moins les faits eux-mêmes que la forme qui leur est donnée. » (Ibid. : 29-30) La science se définit ainsi par le document et contre la littérature précisément contre la rhétorique, c'est-à-dire cet art de persuader par la maîtrise du langage, longtemps au cœur de l'enseignement des humanités et perçu à partir de la fin 
du XIX e siècle comme un « art de parler bien sans penser », selon une formule lapidaire de Gustave Lanson ${ }^{5}$.

Avec cinquante ans de retard, c'est un tel modèle, improprement qualifié de «positiviste », qu'importent Marcel Griaule et Paul Rivet dans l'ethnologie : l'ethnologue est tout sauf un littérateur. Ainsi Silhouettes et graffiti abyssins se donnera pour tâche la "représentation fidèle d'un art abyssin inconnu : celui des jeunes garçons du Godjam ». " Nul souci esthétique n'a présidé à ce travail, précisera Griaule dans l'introduction ; cette publication est strictement scientifique, sans enjolivures.»(Griaule 1933: 5) Dans la Méthode de l'ethnographie, il écrira encore : «Les effets artistiques doivent être l'objet de la plus grande méfiance. » (ME : 83) Dès le retour de sa mission au Godjam, il notait :

«Ce serait singulièrement simplifier le problème que d'essayer de comprendre l'âme de ce grand peuple autrement qu'au travers d'enquêtes superficielles de littérateurs ou de dilettantes. Il serait grand temps qu'on prenne au sérieux les faits sociaux où se révèle cette âme, et qu'on leur fasse l'honneur de les étudier comme une réaction chimique ou un problème de résistance des matériaux. » (Griaule 1930 : 454)

Si l'on en croit la table des matières de la Méthode de l'ethnographie, les cours de méthode que Griaule donne à la Sorbonne à partir de 1943 sont organisés sur le modèle de l' Introduction aux études historiques de Langlois et Seignobos (1898) et semblent clairement inspirés de ce qui fut la "bible» des historiens jusqu'à la fondation des Annales. On y trouve en effet les rubriques suivantes: personnel de la recherche; détection et observation des faits humains; enregistrement; critique des sources. Seul le dernier chapitre, qui ne compte que huit pages, est consacré à « l'exploitation des documents ».

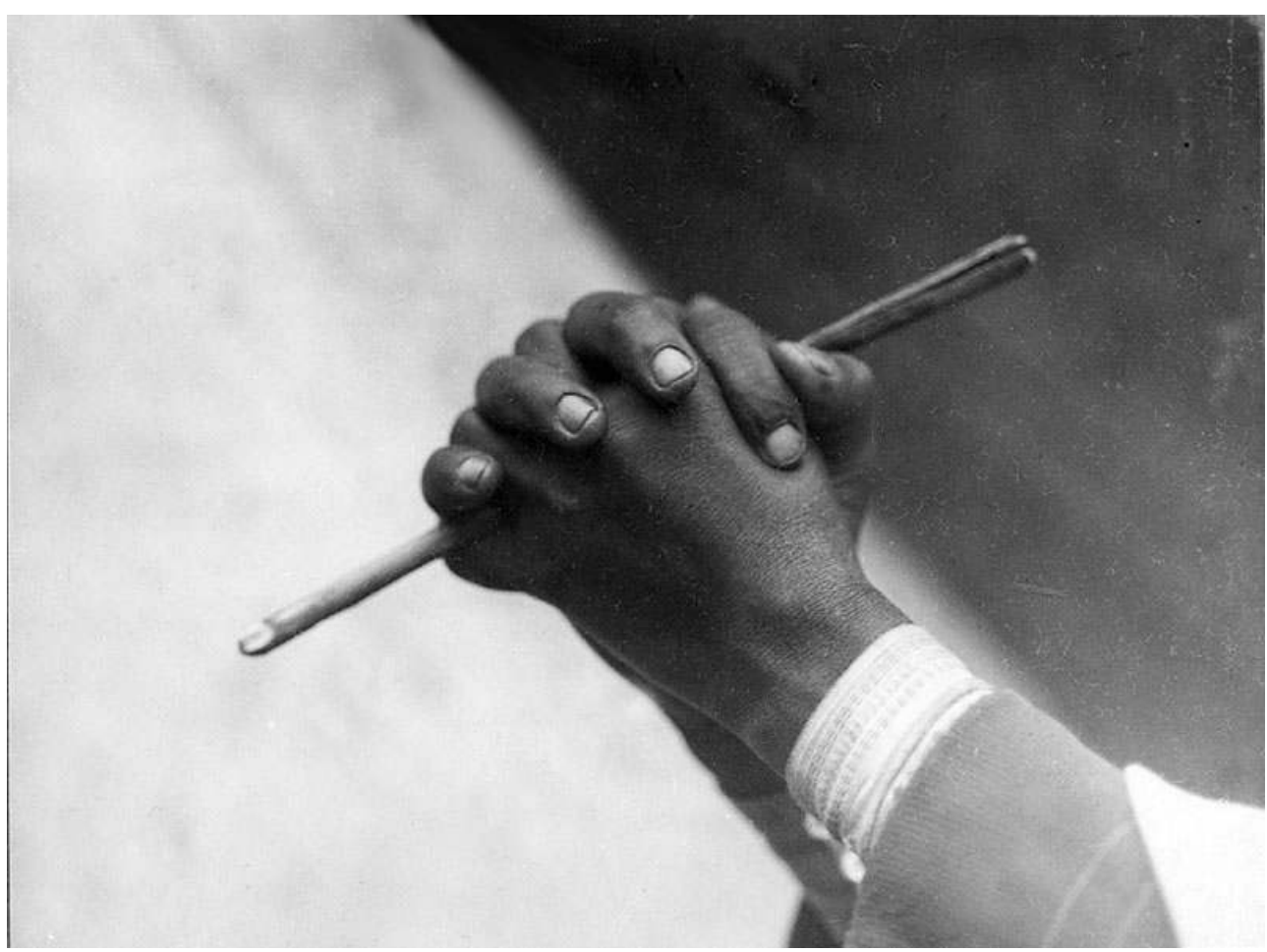

Fig. 7 Jeu de l'oiseau.

(c) musée du quai Branly, photo Marcel Griaule.

Mais précisément, cette réduction de l'épistémologie à une méthodologie passe sous silence les difficultés liées à la restitution et à l'exposé des résultats. Car une fois classé et 
répertorié, le document perd les qualités qu'il avait à l'origine : ce qui était rite, coutume vivante, technique singulière prise dans un ensemble cohérent ne survit pas dans l'archive, dans la fiche ou dans la vitrine du musée. C'est un des autres refrains que l'on trouve sans cesse dans les travaux de Griaule : l'ethnographie est une "science vivante » proposant une "tonique aération des idées». Cette qualité vitale ne subsiste pas cependant lors du retour en France :

« Les fiches, c'est la mort de la fantaisie.» (Griaule 1943 : 9)

«Un à un les objets sont affublés d'un petit numéro et repérés sur le plan. Finie la liberté! Le document est bien englouti, engrené dans la machine à raisonner, valorisé, curieux, exploitable, surgi à la lumière, perdu à jamais pour les morts qui l'avaient laissé là. » (Griaule 1943 : 37)

«[...] dans le camion qui nous emmenait à vive allure vers le sud, nous pensions avec satisfaction aux caisses qui allaient s'accumuler dans les sous-sols du Musée du Trocadéro.

Car, dans ce genre de recherche, tout finit dans des caisses. » (Ibid. : 134) celui-ci ne restitue pas l'atmosphère de la société considérée. D'autre part, il ne se suffit pas à lui-même, car livré tel quel au lecteur ou au visiteur du musée, il ne peut que l'induire en erreur. Autrement dit, le document est inintelligible sans un discours professionnel qui l'encadre et l'interprète. Il y a là un argument essentiel des promoteurs de l'ethnologie : celle-ci doit rectifier les erreurs d'un public menacé par « les formes les plus rétrogrades de l'esprit de troupeau ${ }^{6} »$; il lui faut éduquer les masses, et pour cela, elle ne doit pas proposer un n-ième récit de voyage qui isolerait des faits spectaculaires détachés de leur contexte, mais présenter au contraire des ensembles coordonnés, réinscrire les pratiques curieuses dans un système cohérent. Le musée ethnographique remplira cette fonction édifiante et pédagogique à destination des masses en se construisant certes contre le musée des Beaux-Arts ${ }^{7}$, mais d'abord contre le cabinet de curiosités. Ouvert tard le soir pour que les «travailleurs manuels» et les ouvriers puissent s'y rendre après leur journée de travail, il proposera non seulement des documents, mais aussi des explications rédigées par des professionnels. Le musée de l'Homme aura ainsi une fonction didactique assumée et revendiquée, et la fin des années trente y sera marquée par un véritable activisme pédagogique: conférences, visites guidées, bulletin mensuel Le Musée vivant ${ }^{8}$, etc. À la source de cet activisme, il y a une conviction : le document ne parle pas de lui-même, et il faut en contrôler la réception.

«Un dessin sera joint chaque fois qu'il faudra montrer le maniement de l'objet, un mouvement de la main ou du pied (exemple: pour l'arc et les flèches, il est important de fixer la méthode de lancement par la position des bras, des doigts aux divers moments ; le métier à tisser est incompréhensible sans documents montrant son fonctionnement). » (Mauss $1967: 17$ )

C'est le revers de la scientifisation: lorsqu'une discipline devient science, elle devient aussi inintelligible pour le commun, et elle exige un travail de traduction et de vulgarisation. 
21 Il reste que le défaut essentiel du document n'est pas là. En effet, sans que cela soit articulé à une véritable théorie, il y a une conviction partagée par tous les ethnographes de la période, à savoir que le fait social est un fait moral ou mental. C'est une affirmation développée par Marcel Mauss ou Lucien Lévy-Bruhl, et avant cela par Émile Durkheim, et les étudiants de l'Institut d'ethnologie, qui ne sont pas toujours très au fait des théories sociologiques, ont au moins retenu ceci : chaque société est caractérisée par des façons de penser, des façons de sentir, certaines dispositions affectives particulières. Le terme pour désigner cet ensemble de dis- positions est presque toujours le même : c'est celui d' atmosphère - on trouve l'expression chez Marcel Mauss, chez Alfred Métraux, chez Jacques Soustelle, chez Marcel Griaule, etc. Parfois assorti de l'épithète sociale ou morale, ce substantif permet de désigner le «climat» - Mauss parle également de "tonalité morale »- qui caractérise une société donnée et qui « imprègne » la vie quotidienne, les usages, les rites, etc.

Pourtant, presque tous les ethnographes s'accordent à dire que l'atmosphère ne survit pas dans le document. Le rêve de l'ethnologie des années trente peut ainsi se dire en une formule paradoxale : ce serait un document évocateur ${ }^{9}$, quelque chose qui serait aussi sûr et fiable qu'un document parfaitement établi, mais qui en même temps évoquerait et restituerait ce supplément impalpable qu'est l'atmosphère d'une société. Il existe plusieurs équivalents de ce mythique document évocateur : le document vivant, et surtout le document humain, c'est-à-dire un document qui resterait humain en dépit de l'arrachement à son lieu d'origine. C'est à cette préoccupation que tâchent de répondre les réflexions de Paul Rivet, Georges-Henri Rivière ou André Schaeffner sur la présentation des objets au musée d'ethnographie du Trocadéro rénové, puis au musée de l'Homme : comment faire pour que le document reste humain?

« Toute une technique de la présentation devra intervenir comme suite à la technique de la collecte, si l'on tient à ce que les documents ne deviennent pas de simples matériaux pour une érudition pesante et ne se dépouillent pas de tout contenu humain, eux qui n'avaient de plus grand intérêt que, précisément, cette qualité d'être des choses "humaines".

[...] au musée de l'Homme, la confrontation [des différents objets, des photographies, des squelettes et des crânes] correspond avant tout à une volonté de ne pas abstraire les objets des groupes humains qui les ont produits, non plus que l'inverse. Ainsi [...] ces humanités mêmes - bien qu'à l'état d'ossements - retrouvent une sorte de paradoxale vie, d'être présentées à proximité de ce qui exprime concrètement leur existence sociale. » (Leiris 1938 : 345)

Griaule lui-même constate :

« L'objet n'est en effet qu'une phase très restreinte de vastes activités, et c'est une piètre victoire que la récolte de ces témoins muets s'ils ne peuvent être remis, grâce à une documentation intensive, dans l'atmosphère de la société qui les a produits. " (ME : 29)

On le voit, la réponse de l'auteur des Flambeurs d'hommes est volontariste : si le document ne restitue pas l'atmosphère, c'est qu'il faut en ajouter d'autres...

Mais à la toute fin de la Méthode de l'ethnographie, un doute semble assaillir Griaule, alors qu'il aborde en quelques pages rapides la dernière phase du travail, l'« exploitation des documents ». Dans ce bref chapitre, il fait quelques concessions inattendues. Malgré le refus affiché de "l'enjolivure" esthétique, Griaule admet que «lorsqu'il expose les résultats de ses travaux, l'ethnographe ne fait pas que décrire » : 
« Il est [...] indispensable de présenter au lecteur les faits dans leurs détails mêmes [...] l'écrivain ne s'interposant que pour un minimum d'élaboration. Inversement, $d u$ point de vue de l'atmosphère dans laquelle se sont déroulés les faits [je souligne], l'écrivain a le loisir d'introduire des impressions, des incidents subjectifs qui seront des plus utiles. C'est qu'en effet, s'il s'agit d'un rite, par exemple, cette activité toujours spectaculaire comporte une gesticulation et une formulation obéissant à des règles esthétiques diffuses, provoquant des réactions esthétiques précises. Il ne serait pas exact de reproduire, même très fidèlement, cette gesticulation et cette formulation sans leur rendre par des moyens également esthétiques leur aspect réel. » (ME : 103)

Il est donc des faits pour lesquels la « reproduction ", " même très fidèle ", n'est tout bonnement pas " exacte"; dans le cas du rite, des "règles esthétiques » suscitent des « réactions esthétiques » qui exigent des « moyens esthétiques ». Griaule poursuit :

«On se trouve donc placé devant deux nécessités contradictoires. L'écrivain doit disparaître quand il s'agit d'exposer la marche d'un rite, et d'autre part, il devra faire appel à toutes les ressources de sa personnalité pour rendre l'atmosphère du rite. Dans le premier cas, il emploiera un style froid, voire plat; il sacrifiera tout effet littéraire à la précision. Il devrait s'inspirer du style du Code civil ou de l'article d'encyclopédie [...]. Dans le second cas, il devra être bon littérateur [je souligne]. » (ME : 103-104)

Tel est donc le paradoxe : d'un côté, l'ethnologie est une science qui se construit contre « les dilettantes et les littérateurs »; de l'autre, elle exige des spécialistes qu'ils soient de « bons littérateurs ».

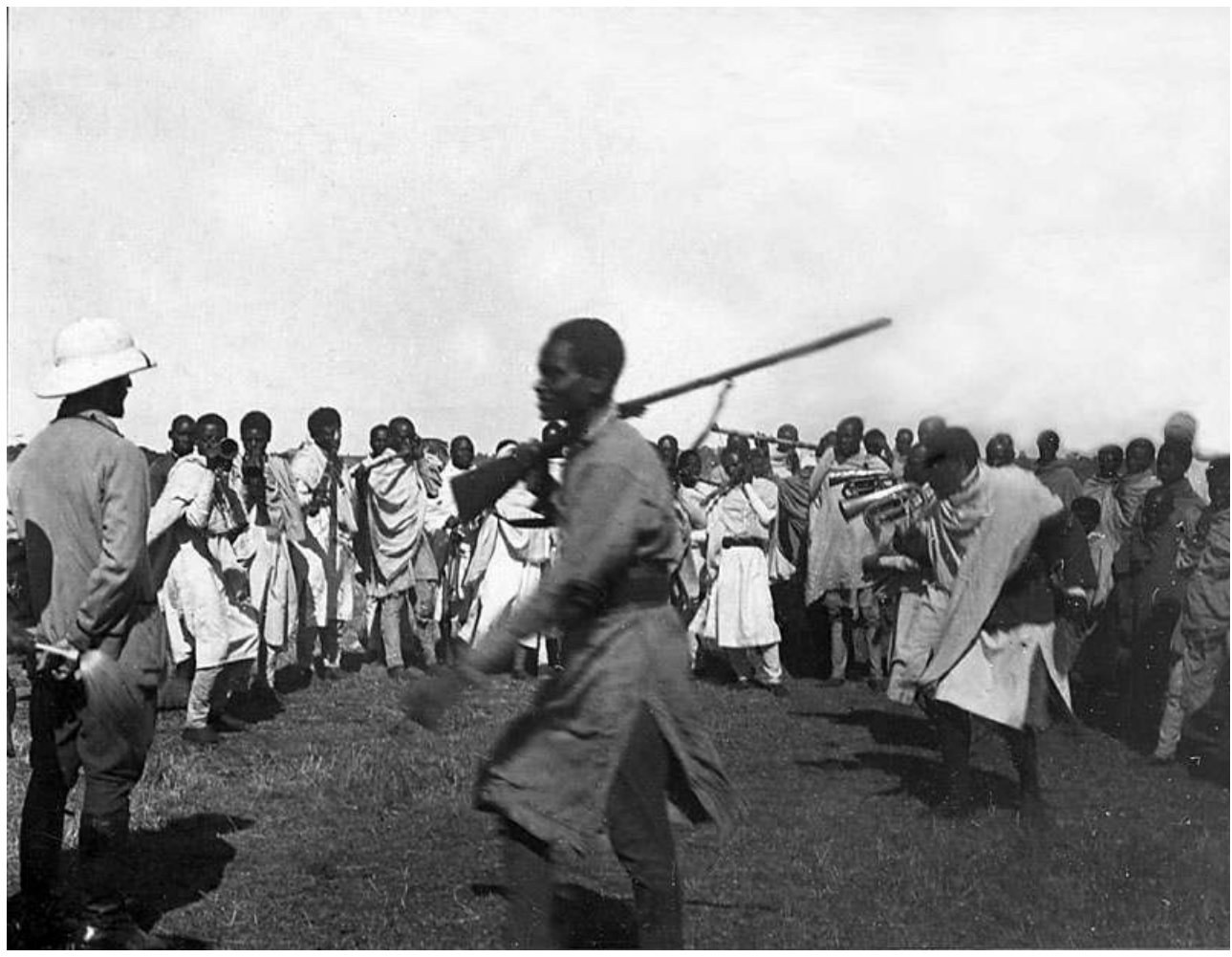

Fig. 8 Musiciens du ras Haïlou donnant une aubade.

(c) musée du quai Branly, photo Marcel Griaule. 


\section{Les Flambeurs d'hommes ou l'impossible document humain}

Les Flambeurs d'hommes doit d'abord être compris dans le cadre de ce paradoxe : l'ouvrage vise de toute évidence à compenser le travail mortifère de la science et l'arrachement du document à son lieu; il se veut une tentative de restitution de l'atmosphère de la vieille société éthiopienne, une de ces réalités " pour [lesquelles] il n'existe pas encore d'autres moyens d'expression que le style». Mais une difficulté surgit d'emblée: comment restituer l'atmosphère sans être taxé de faire de la "littérature " ? Sans être soupçonné de céder à la rhétorique et aux plaisirs de la belle forme ou du beau langage ? Car la rhétorique, c'est précisément cela : l'art de faire sentir par le langage, de susciter chez le lecteur ou l'auditeur un état - pathos - par un travail sur le logos. Griaule est ainsi pris dans une contradiction : d'un côté, le document ne suffit pas ; de l'autre, la rhétorique est interdite.

Il faut ici se demander ce qu'est un document dans la perspective de Griaule (et de l'histoire méthodique). On pourrait en donner la définition suivante : un document est un énoncé (ou une image) dont la production n'est pas déterminée par la réception savante. Si un acte notarié est un document historique fiable, c'est qu'il n'a pas été rédigé en vue de sa réception par l'historien. De la même façon, pour l'ethnographe, un document fiable, c'est par exemple un mythe, dont il sait qu'il n'a pas été embelli parce qu'il l'écoutait, un rite dont il sait qu'il n'a pas été modifié parce qu'il était présent, etc. À l'inverse, le " littérateur ", c'est le pseudo-historien ou le pseudo-ethnographe dont le discours n'est pas digne de confiance parce qu'il se soucie de son public, parce qu'il soigne ses effets... Les Flambeurs d'hommes correspond à une tentative de résolution de ce dilemme: Griaule va inventer un document évocateur, c'est-à-dire un texte qui fera sentir les façons de sentir, mais qu'on ne pourra pas soupçonner de rhétorique, d'affectation ou de souci de plaire - 
autrement dit, un texte qui communique l'atmosphère, mais qui la communique à son insu

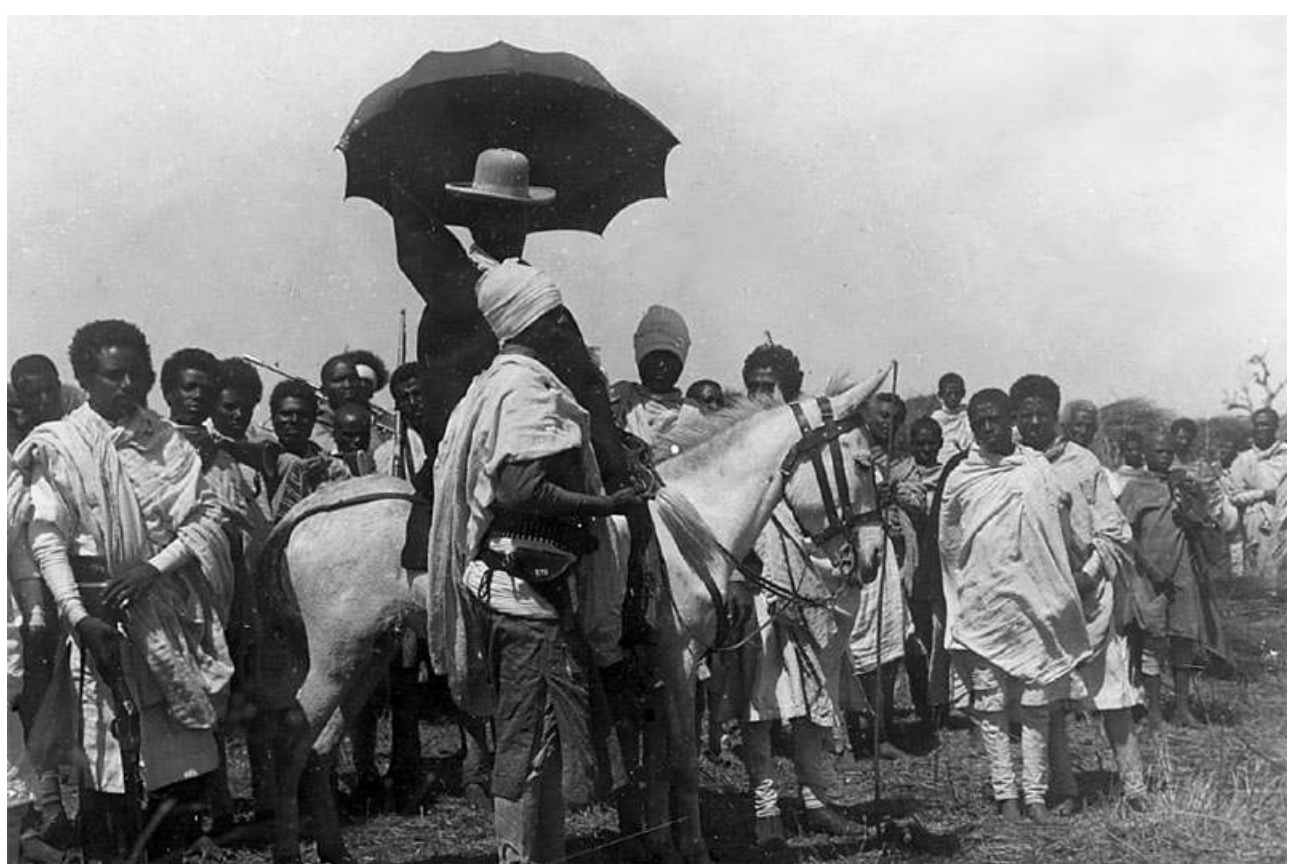

Fig. 9 Le ras Haillou et sa suite.

(c) musée du quai Branly, photo Marcel Griaule.

31 Ce n'est pas inimaginable ; il en existe un modèle célèbre dans la littérature, ce sont les Chroniques italiennes de Stendhal. En 1833, alors qu'il occupe le poste de consul de France à Civitavecchia, Stendhal découvre à Rome, au palais Cateani, une série de vieux manuscrits datés essentiellement du xvie siècle ; ceux-ci relatent certains épisodes sanglants nés des rivalités entre différentes familles de l'aristocratie italienne. Il décide de faire copier, puis de traduire ces manuscrits, et projette de les réunir dans un recueil d'« historiettes romaines » qui devrait permettre, selon lui, de mieux comprendre «ces mœurs qui ont enfanté les Raphaël et les Michel-Ange ${ }^{10}$ ". L'entreprise de Stendhal répond à un projet de connaissance qu'on pourrait presque dire ethnologique avant la lettre: il s'agit de comprendre "de l'intérieur" cette époque qui a produit à la fois César Borgia et Benvenuto Cellini, saisir ce paradoxe qui veut que la même civilisation ait engendré à la fois les plus grandes productions du génie humain et les crimes les plus sanglants. Outre qu'elle déforme les faits, la " grande histoire », en s'intéressant en priorité aux papes, aux grandes familles florentines et à la diplomatie, manque un objet essentiel : les « mœurs », les « caractères ", les « façons de sentir ». Les romans de l'époque (ceux de George Sand et d'Ann Radcliffe au premier chef) ne sont pas davantage capables de restituer « cette façon passionnée de sentir qui régnait en Italie vers 1559 », car ils sont corrompus par la «vanité littéraire » et le goût de la couleur locale (Stendhal 1973 : 85). À l'inverse, si les chroniques découvertes au palais Cateani sont fiables, c'est qu'on n'y trouve nulle affectation et nul souci de plaire. Les conventions du genre autant que son inscription sociale (un chroniqueur attitré est chargé de recueillir les faits et gestes de telle ou telle famille de la noblesse) le mettent à l'abri de tout soupçon de mise en scène. Puisque le chroniqueur ne doit pas quitter sa position de « rapporteur ", il n'est pas suspect de céder aux tentations de la "vanité littéraire ». Dès lors, le style joue comme un révélateur des 
sensibilités du temps, et les chroniques proposent un «calque fidèle des façons de sentir du Xvie siècle ${ }^{11} »$ (ibid.).

Malheureusement, Griaule n'a pas eu la chance de tomber sur une chronique éthiopienne. Comme ce document n'existe pas, il va donc l'inventer. Pour faire sentir les façons de sentir, pour restituer le caractère singulier de la nation abyssine, il va imaginer une chronique éthiopienne. Bien sûr, on n'invente pas un document; un tel projet est contradictoire, et Griaule ne formule jamais les choses de la sorte, mais c'est une hypothèse qui permet de rendre compte de l'opacité de l'ouvrage : il faut lire le livre de 1934 comme une chronique écrite par un lettré éthiopien. Certes, c'est le récit de l'expédition de Griaule, mais c'est un récit raconté « du point de vue » d'un Abyssin, afin de rendre mais sans rhétorique, sans risque d'être taxé de littérature, et comme par inadvertance la noblesse des paroles et des attitudes, une religiosité partout présente, le goût de la belle langue et des «sublimes phrases ». On peut par exemple considérer à nouveau le passage déjà cité, consacré à l'altercation entre Griaule et ses porteurs : « il y avait eu des coups donnés par le Blanc et non rendus, car un Blanc est toujours homme de gouvernement, qui fait éclater des complications dès qu'on le touche. » Cet épisode a suscité chez plusieurs commentateurs des réactions effarouchées (non seulement Griaule frappe les caravaniers, mais en plus il le raconte et, comble de l'outrecuidance, à la troisième personne...). Cependant, la singularité du passage ne tient pas d'abord à une scandaleuse héroïsation de la brutalité (du reste, s'il y a un héros ici, c'est plutôt «l'homme de poids » qui répond à Griaule en mêlant sagesse et sens de la formule), mais plutôt à la bizarrerie du mode énonciatif, bizarrerie qui se résout si l'on imagine un scribe éthiopien relatant la scène à laquelle il a assisté, et présentant « objectivement » le conflit d'intérêts, cédant certes devant la force, mais conservant par-devers lui ses convictions :

«Le Blanc, en effet, ne connaît pas le fond des choses. Pour lui un fleuve est un fleuve, c'est-à-dire des causes de soucis pour les bagages et un prétexte à vérifier les chargements. Pour lui, les questions principales sont la force du courant et la profondeur du gué. » $(\mathrm{FH}: 37)$

On peut lire, avec la même hypothèse, le récit du passage du gué du Nil qui apparaît comme une scène d'histoire médiévale, racontée sur un ton déclamatoire traduisant «innocemment» le prestige qui auréole une telle action d'éclat aux yeux des «Abyssins »:

« La caravane s'ébranla solennellement [...].

Tous les échos du gué répétèrent mille et mille fois l'honorable nouvelle que des Francs et leurs biens s'avançaient lentement dans les eaux du Nil. Au nombre des coups de feu, chacun apprit que ces biens étaient grands et les étrangers considérables, puisqu'on ne regardait pas à la dépense pour honorer les lieux.» (FH : 46)

Le récit du supplice de la mort par la mousseline s'éclaire lui aussi, s'il est lu dans cette perspective :

«[...] le supplicié jeta son premier hurlement, et [...] s'échappa comme un lévrier dans un cercle formé immédiatement par les personnes suivantes:

Chef d'avant-garde Alameurrao, chef du Damot, décoré de l'ordre de Salomon, vingt-cinq dépouilles viriles, cinq éléphants, dix lions, trois rhinocéros.

Chef d'avant-garde Chambal, chef de l'Atchefer, décoré de l'ordre godjamite de Théodoros, quinze dépouilles viriles, trois éléphants, dix lions.

Chef d'aile droite Chaffarao, chef des Goumz, trente dépouilles viriles, cinq buffles. Chef d'aile droite Engueda Work, dit «le Ravageur», chef des Abigars, quarantecinq dépouilles viriles. » $(\mathrm{FH}: 125)$ 

chronique des hauts faits du ras Haïlou. De même, les asyndètes, les sentences obscures (" le Nil est cette chose qui empêche le caravanier de dormir », "siffler c'est pactiser avec les rats ») et la plupart des opacités - les toponymes (« Abbaye-de-Marc », « le plateau de Saint-Jean-Venteux », etc.), le choix de références visiblement empruntées à l'univers symbolique des Éthiopiens du lac Tana («au treizième jour du mois de guennbôte ») fonctionnent comme des garanties d'authenticité: elles signalent l'inscription éthiopienne d'une narration écrite sans souci du destinataire et conformément à un univers moral dont les codes n'ont pas à être explicités.

De là, donc, la troisième personne : Griaule n'est pas un je-auteur, mais «le Blanc », et la narration est déléguée à une instance anonyme et insaisissable, censée restituer une perception « éthiopienne » des faits. Pour les mêmes raisons, la photographie est absente. L'adjonction de clichés aurait d'abord doté d'un poids de réalité et de modernité un livre dont l'objectif le plus clair est de faire revivre une Éthiopie idéalisée ; en introduisant des éléments trop évidemment référentiels, elle aurait contredit un texte qui, en dehors de l'avant-propos, ne cesse d'effacer sa propre inscription dans l'espace et dans le temps. Mais surtout, elle entrerait en contradiction avec cette fiction de chronique à l'éthiopienne. Dans le récit de voyage, en effet, la photographie a avant tout valeur d'attestation, mais cette attestation a pour revers l'inscription rhétorique d'une première personne s'adressant à son lecteur ; c'est le "j'y suis allé » analysé par Clifford Geertz comme fondement de la position d'autorité de l'ethnographe (Geertz 1996 [1988]). Or Griaule évite précisément la première personne, et la photographie s'intégrerait mal à un tel dispositif. Elle témoignerait en effet d'une transitivité et d'un projet de communication incompatible avec une "description objective", qui ne doit pas être suspecte d'affectation et qui, comme la chronique «Vittoria Accoramboni » de Stendhal, n'aura le droit d'être pittoresque qu'à son insu. Ici encore, le document évocateur communique, mais il communique comme par mégarde et contre son gré.

Pourtant, nul n'ignore que Griaule est l'auteur des Flambeurs d'hommes. Lui-même ne s'en cache pas, et c'est bien son nom sur la couverture du livre. Il faut donc inverser la question : pourquoi n'avoir pas poussé le subterfuge jusqu'au bout? N'avoir pas signé le livre d'un pseudonyme éthiopien? Pourquoi Griaule ne peut-il pas renoncer à son statut d'auteur? Parce que, précisément, même le document évocateur ne parle pas de lui-même; il faut un savant pour en encadrer la réception. On touche ici du doigt la contradiction la plus profonde à laquelle Griaule est confronté, celle qui est proprement indépassable et qui rend certains passages des Flambeurs d'hommes littéralement incompréhensibles: quand bien même un Éthiopien lettré aurait relaté sous forme de chronique les péripéties de Griaule en Abyssinie, le lecteur ne pourrait la lire et la comprendre sans explications adventices, de la même façon que le visiteur du musée doit disposer d'une photographie du métier à tisser pour en comprendre l'usage et la fonction. Il faut donc que Griaule parle en son nom, en tant que savant: c'est ce qu'il fait dans l'avertissement, et c'est pourquoi il doit signer le livre.

"Descendre les pentes orgueilleuses de l'érudition ", "présenter au plus grand nombre de lecteurs un ouvrage lisible»: ces justifications sont très curieuses et, de toute évidence, ne correspondent pas à la relation entre Jeux et divertissements abyssins et Les Flambeurs d'hommes ; il n'y a aucun rapport de vulgarisation de l'un à l'autre, mais Griaule doit préciser que c'est en tant qu'ethnographe professionnel qu'il écrit et qu'il sait de quoi il parle. De là la nécessité d'une préface écrite, elle, à la première personne et qui 
souligne la compétence de l'énonciateur, compétence acquise à la fois auprès de ses pairs savants, "spécialistes » et "érudits ", et au prix d'une enquête qui l'a rendu familier d'une « terre amie, parente, dont [il connaît] la langue et l'esprit, dont [il connaît] par leurs noms les arbres, les rivières et les démons » (FH : 28).

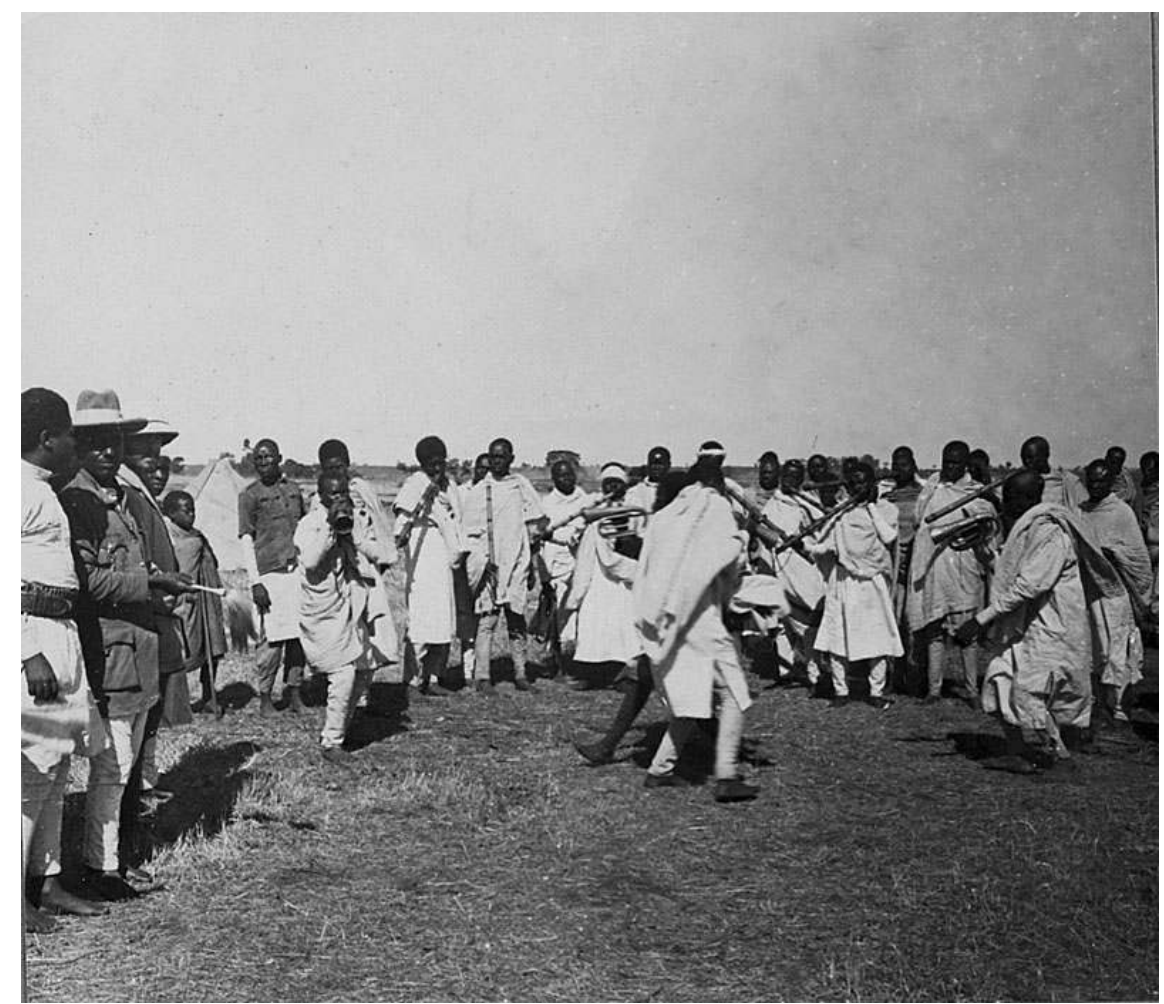

Fig. 10 Musiciens du ras Haïlou.

(c) musée du quai Branly, photo Marcel Griaule.

Si Les Flambeurs d'hommes est un livre aussi étrange et aussi inégal, c'est d'abord que son statut énonciatif est instable et qu'il hésite entre la chronique déclamatoire "à l'éthiopienne » et le récit ethnographique traditionnel, rédigé par un savant qui raconte son expédition pour un public européen (mais à la troisième personne, ce qui, à la lecture, produit un effet curieusement discordant). La narration est bizarrement flottante; on change de point de vue, passant de la perception censément éthiopienne des faits à l' évocation suggestive à destination du lectorat français, ce qui crée de brutales ruptures de ton et de singulières dissonances. Ainsi, au milieu du récit très solennel de l'emmaillotement préparant l'immolation du condamné, on trouve cette phrase, de toute évidence « adressée » à un lecteur français : « l'ensemble rappelait une armure du Moyen Âge, ou un bonhomme Michelin sans bras. » (FH : 123)

Il arrive même que la contradiction entre les deux registres devienne intenable, et que l'opacité du texte atteigne un degré tel que sa lisibilité même semble remise en cause. Ainsi de la description des poèmes récités par «l'hermaphrodite de la cour » du ras Hailou :

«Il y avait dans ces quelques exclamations une poésie inextricable pour des cervelles étrangères. Tous les assistants hochaient la tête en retenant leur souffle. Et ce n'était pas une mince raison de modestie pour les Francs aux puissantes inventions mécaniques et pères des armes à feu, que de se sentir si seuls, et si privés $\mathrm{du}$ sens profond des choses, dans cette multitude aux émotions millénaires. » (FH : 88) 
41 En première analyse, le passage semble relever de la narration "à l'éthiopienne "; une formule comme « les Francs aux puissantes inventions mécaniques et pères des armes à feu » semble attribuable à un chroniqueur lettré. Pourtant, dans le même temps, le savant prend la parole: il est question d'une "poésie inextricable pour des cervelles étrangères ». Mais l'expérience $d u$ «Franc-savant» relatée est précisément une expérience d'incompréhension qui l'invite à la modestie et à l'humilité : il est «privé du sens profond des choses "... D'un côté, le narrateur comprend en tant qu'Éthiopien la poésie de cour abyssine ; de l'autre, en tant que savant européen, il comprend que cette poésie est incompréhensible pour des cervelles étrangères ; mais d'un troisième côté, il raconte que le Franc - c'est-à-dire lui-même - ne comprend pas. Autrement dit, il comprend et ne comprend pas en même temps.

Cette contradiction énonciative locale cristallise en fait une contradiction beaucoup plus fondamentale, et montre que l'épistémologie implicite de Griaule le pousse à des compromis intenables: il voudrait à la fois établir la fiabilité du document et en compenser les insuffisances sans faire appel à la rhétorique. Mais la littérature du «bon littérateur » qu'il réclamera dans sa Méthode de l'ethnographie ne requiert pas seulement le mariage de l'évocation et de la connaissance, de la puissance suggestive et de la vérité scientifique ; elle suppose aussi une confiance dans l'exercice du jugement du lecteur. Or cette confiance, il ne peut la lui accorder, puisqu'en se constituant comme une science, l'ethnologie s'est coupée du commun, elle est devenue un domaine d'expertise auquel le public ne peut plus accéder sans la médiation du professionnel. En écrivant Les Flambeurs d'hommes, Griaule prétend donc produire un document évocateur, c'est-à-dire un texte qui, d'une part, soit intégralement fiable et échappe au soupçon d'affectation (d'où l'absence de photographie et de première personne) et qui, d'autre part et pour cette raison même, révèle l'atmosphère morale de l'Éthiopie septentrionale à des lecteurs qui ne la connaissent pas et pour lesquels, en tant que savant, il veut bien « descendre les pentes orgueilleuses de l'érudition ». Le subterfuge du récit à la troisième personne correspond à ce projet intenable et contradictoire : celui d'un voyageur qui communique à la fois en son nom et à son insu.

43 Les Flambeurs d'hommes peut ainsi être lu comme le produit des tensions entre littérature et ethnologie au moment où la discipline s'institutionnalise, tensions qui, dans le récit de Griaule, se trouvent à l'état presque cristallisé. Lorsqu'on évoque les relations entre les deux ordres dans les années trente, on pense d'abord à L'Afrique fantôme ou au compagnonnage de l'avant-garde post-surréaliste et des ethnographes du musée du Trocadéro autour de la revue Documents. Mais avant même ces échanges, les références à la littérature qu'on trouve dans les textes ethnologiques relèvent d'une conception toute « classique », pour ne pas dire conservatrice, de la littérature, à mille lieues de celle des surréalistes (qui, d'ailleurs, n'emploient jamais le mot que par antiphrase) : la littérature est désirable pour sa capacité d'évocation; elle est la technique de traduction et de restitution des réalités morales ${ }^{12}$. Qui plus est, elle est, par essence, l'affaire de tous; discours non spécialisé, il n'est pas un homme de bonne volonté qu'elle ne saurait toucher. Mais dans le même temps - et la plasticité du terme autorise ce déplacement -, la "littérature » est ce dont il faut se déprendre; elle est le lieu où règnent l'abus du langage figuré, les séductions de l'imagination et de la rhétorique - autant de traits d'une connaissance préscientifique -, ce qui fait d'elle à la fois, donc, le contraire et le passé de la science. 

sans encourir le soupçon de littérature? Au nom de l'éducation des masses. Le prétexte de vulgarisation est la seule façon de rendre pensable une exigence de rhétorique dans le cadre d'une science documentaire. Littéralement, la vulgarisation autorise la littérature là où elle est habituellement interdite, et permet, sinon de résoudre, en tout cas de supporter les tensions engendrées par l'élaboration de l'ethnologie, science des réalités morales, sur un modèle « positiviste » et anti-rhétorique. On pourra d'ailleurs noter que nombre de controverses contemporaines touchant l'« excès " de littérature dont serait victime tel ou tel ouvrage ethnographique s'inscrivent encore dans cette configuration problématique qui était déjà celle des premières années de la discipline ${ }^{13}$.

configuration, donc, n'est pas propre à Griaule, il y occupe toutefois une position originale. Celle-ci tient d'abord à une conception particulièrement extensive de l'autorité et des prérogatives du savant, puisque la compétence acquise dans le cadre de la science continue à s'exercer en dehors d'elle et vaut comme un blanc-seing pour l'évocation « littéraire » et "grand public », au mépris même de l'exigence de véracité. Comme il a « sué sur des selles indigènes » ( $\mathrm{FH}: 143)$, comme ses pairs l'ont « classé au premier rang des jeunes africanistes » $\left(\mathrm{FH}: 25^{14}\right)$, Griaule ne craint pas d'insérer dans la narration des événements imaginaires, de déclarer, contre l'évidence, que Les Flambeurs d'hommes n'est pas un "récit romancé », et de présenter comme une "description objective » un texte qui comporte nombre d'affabulations ${ }^{15}$, au prétexte - on peut le supposer - qu'à ses yeux de tels épisodes exemplifient «l'esprit » de la société « abyssine » mieux encore que des faits qu'il aurait pu observer. Lui qui donne constamment des leçons d'humilité à son public, invitant à la "modestie » les «Francs » qui le lisent, en manque singulièrement dès lors qu'il s'agit d'affirmer sa propre compétence.

Dans les préambules des différentes Chroniques italiennes, Stendhal explique la nécessité de se cantonner au rôle de traducteur, car un « jeune Français » de 1838 ne peut être «bien sûr de deviner ce qu'éprouvaient ces âmes italiennes de l'an 1559 ». Mais Griaule ne connaît pas de tels doutes, puisqu'il parle de contemporains et qu'il a franchi la distance géographique et culturelle qui le séparait d'eux; il ne lui restera qu'à imaginer la chronique. D'autres ethnographes de la même génération, plus scrupuleux, s'interrogent à chaque étape du travail : lors de l'enquête elle-même, aux différents stades de l'écriture, au moment de la restitution. Moi, jeune Français de 1934, suis-je bien sûr d'éprouver ce qu'éprouvent ces Lacandon dont je partage les jours ; suis-je bien sûr de pouvoir traduire ces sentiments ; suis-je même bien sûr de les retrouver en moi-même deux ans après mon retour; et surtout, suis-je bien sûr de les faire sentir à d'autres? Telles sont, par exemple, les interrogations qui traversent Mexique, terre indienne de Jacques Soustelle (1994 [1936]). Griaule les congédie toutes d'un seul mouvement. Protégé des incertitudes par la substantialisation des populations qui est au principe de son travail, il est convaincu qu'une enquête bien conduite pourra épuiser le donné. Il n'y a pas d'insuffisance qui ne puisse être palliée par un complément d'information: on pourra toujours " "boucher les trous" par une enquête ultérieure " (ME : 87). De la même façon, il ne s'embarrasse pas avec le problème de l'ineffable; s'il est "bon littérateur", il parviendra toujours à « rendre l'atmosphère » (selon les termes de la Méthode d'ethnographie) car, pour le reste, il ne doute à aucun moment d'avoir saisi « l'âme de ce grand peuple » (Griaule 1930 : 454).

Mais cette affirmation d'autorité a une autre conséquence; elle conduit à un séparatisme exclusif entre le savant et ses lecteurs. Telle est sans doute la différence fondamentale entre l'entreprise de Griaule dans Les Flambeurs d'hommes et le projet anthropologique de 
Stendhal dans les Chroniques italiennes (nonobstant, bien sûr, les différences esthétiques et thématiques). Outre que Stendhal dispose de ces documents évocateurs qui font défaut à Griaule, il n'a pas perdu tout espoir d'une communication fondée sur une communauté de nature entre auteur, sujet et lecteur. Les Italiens du xvie siècle se sont certes éloignés de nous et de "notre siècle sans préjugés ", mais pas au point de devenir incompréhensibles aux gens de bien; le happy few - ceux que Stendhal appelle, dans "Les Cenci», ses "lecteurs de bon goût" - saura saisir cette réalité morale traduite dans le style des chroniques. Malheureusement, l'ethnologie apparaît au temps des masses, et elle ne cesse de répéter que le document ne parle pas de lui-même. D'abord, parce que concrètement, il est réservé aux spécialistes, ensuite parce qu'il ne transmet pas l'atmosphère, enfin et surtout parce que, livré brut à un grand public abreuvé de clichés exotiques, il ne peut qu'induire en erreur, et il faut l'arsenal de la science pour en contrôler la réception.

Ici encore, on voit que l'utopie du document évocateur ne suppose pas seulement l'alliance entre la puissance suggestive du style et la vérité scientifique; elle exige qu'un certain crédit soit accordé au lecteur, et que soit postulée une communauté, au moins relative, entre l'auteur et son public. Or, dans tous ses écrits, Griaule ne cesse d'affirmer sa singularité et de marquer sa différence: avec les voyageurs non professionnels, «dilettantes et littérateurs » invités à "rentrer en eux-mêmes » (FH : 139); avec les anthropologues de cabinet et autres érudits, "explorateurs de bibliothèques " et "chasseurs de virgules» (FH: 143); avec ceux-là mêmes qui le consacrent comme " africaniste » puisqu'il ironise contre "[son] maître Marcel Mauss » dont les conseils s'avèrent parfois inadaptés à l'enquête (FH : 93); avec surtout son public, inconscient de la relativité des mœurs qui sont les siennes et constamment invité à la modestie et à l'humilité.

Dans les années trente, Griaule pousse très loin le souci de s'excepter de son lectorat, et tous ses écrits manifestent comme une angoisse d'être saisi et situé. Il flotte ainsi, inassignable, jouant sans cesse et selon les contextes la théorie contre le terrain ou le terrain contre la théorie, l'ici contre l'ailleurs ou l'ailleurs contre l'ici. Mais dans Les Flambeurs d'hommes, la question de l'énonciation (qui parle?) n'est plus seulement habilement évitée ou déniée, elle est littéralement forclose. L'ouvrage de 1934 incarne ainsi une tentation sans doute consubstantielle à une ethnologie toujours partagée entre « ici » et « là-bas », tentation qui consiste à ériger non le discours de l'ethnographe, mais sa personne même en lieu unique - et vide - de réconciliation de l'hétérogène. Ce n'est que bien plus tard, à la fin de sa vie, alors que la reconnaissance de la discipline pour laquelle il avait tant lutté sera enfin acquise, que Griaule osera énoncer les «nécessités contradictoires » auxquelles l'ethnographe est confronté, partagé qu'il est entre le «sacrifice de tout effet littéraire à la précision » et les exigences du «bon littérateur ». Mais à peine aura-t-il évoqué «la complexité des problèmes qui se posent au rédacteur ", que l'auteur de la Méthode de l'ethnographie se repliera, comme à l'habitude, sur sa position préférée, celle de l'ethnographe aux « jugements à tête froide » qui renvoie dos à dos l'ici et l'ailleurs et refuse de trancher. Il mourra avant de pouvoir écrire une conclusion. Son cours sera publié un an plus tard, en 1957, par les soins de sa fille, mais depuis quelques années déjà, l'ethnologie est entrée dans une nouvelle phase de son histoire, et les interrogations de Griaule arrivent à la fois trop tard et trop tôt: trop tard, car elles témoignent d'une conception substantialiste des cultures qui est en train de se défaire de toutes parts sous l'effet des revendications des populations colonisées ${ }^{16}$; trop tôt, car ces réflexions (embryonnaires) sur l'écriture de l'ethnographie ne pouvaient guère trouver 
d'écho en une période où les sciences de l'homme croyaient avoir enfin trouvé dans le structuralisme une issue aux impasses de la science documentaire.

\section{BIBLIOGRAPHIE}

Cohen, Marcel

1962 « Sur l'ethnologie en France », La Pensée 105, octobre : 85-96.

Debaene, Vincent

2006 « "Étudier des états de conscience", la réinvention du terrain par l'ethnologie (1925-1939) », L'Homme 179 : 7-61.

Geertz, Clifford

1996 [1988] Ici et là-bas. L'anthropologue comme auteur, trad. Daniel Lemoine. Paris, Éditions Métailié.

Griaule, Marcel

1930 « Une mission ethnographique et linguistique en Éthiopie (1928-1929) », L'Afrique française, août : 453-454.

1933 Silhouettes et graffiti abyssins. Paris, Larose.

1935 Jeux et divertissements abyssins. Paris, Ernest Leroux.

1943 Les Saô légendaires. Paris, Gallimard.

1951 « Connaissance de l'homme noir », inGriaule et al., La Connaissance de l'homme au XX siècle. Neuchâtel, La Baconnière : 147-166.

1957 Méthode de l'ethnographie. Paris, PUF.

1991 [1934] Les Flambeurs d'hommes. Paris, Berg International.

Jolly, Éric

2001 « Du fichier ethnographique au fichier informatique. Le fonds Marcel Griaule et le classement des notes de terrain ", Gradhiva 30-31: 81-103.

Lanson, Gustave

1964 [1902] « Contre la rhétorique et les mauvaises humanités », Essais de méthode, de critique et d'histoire littéraire. Paris, Hachette : 57-60.

Leiris, Michel

1938 « Du musée d'Ethnographie au musée de l'Homme », La Nouvelle Revue française, août :

344-345.

Mauss, Marcel

1967 [1947] Manuel d'ethnographie. Paris, Payot.

Mercier, Jacques 
1988 Asrès, le magicien éthiopien. Souvenirs 1895-1985. Paris, J.-C. Lattès.

1993 « Rencontres, fiches et savoir. Le butin des zar », Gradhiva 14 : 41-49.

Monod, Gabriel

1876 « Introduction. Du progrès des études historiques en France depuis le XVI ${ }^{\mathrm{e}}$ siècle ", Revue historique, année I, t. 1, janvier-juin : 5-38.

Soustelle, Jacques

1994 [1936] Mexique, terre indienne. Paris, Hachette (« La Vie quotidienne »).

Stendhal

1973 Chroniques italiennes. Paris, Gallimard (« Folio »).

\section{NOTES}

1. Les fichiers de Griaule sont conservés à la bibliothèque du Laboratoire d'ethnologie de Nanterre : on y trouve 20000 fiches classées alphabétiquement par thèmes (avant la guerre), puis par régions. Voir Fonds Marcel Griaule (en collaboration avec Jacques Mercier et Marie-Dominique Mouton), brochure éditée par le Laboratoire d'ethnologie et de sociologie comparative (université de Paris X-Nanterre), 1997. Voir Jacques Mercier 1993 et Éric Jolly 2001.

2. Voir sur ces différents points la présentation de Michel Perret qui précède la réédition du livre aux éditions Berg International (1991). C'est à cette réédition, préfacée par Geneviève CalameGriaule, que renvoie dans la suite le sigle FH suivi d'un numéro de page. Le sigle ME renvoie quant lui à la Méthode de l'ethnographie publiée de façon posthume aux PUF en 1957, à partir des notes des cours délivrés par Griaule à la Sorbonne.

3. Jacques Mercier note que l'épisode «fut considéré comme authentique par nombre d'historiens », mais qu'il s'agit d'une déformation « d'un méfait de jeunesse du "bon" Ménélik qui avait châtié de cette façon un rebelle nommé Bezzabeh » (Mercier 1988 : 281).

4. À noter que les traductions anglaise (Abyssinian Journey, 1935) et américaine (Burners of men, 1935) comporteront, quant à elles, seize planches de photographies.

5. «Les humanités qui ont fait un Rabelais et un Montaigne se rapetissent à la rhétorique. Ce n'est même plus un art de penser qu'on y prend, mais un art de parler bien sans penser. » (Lanson 1964 [1902] : 58)

6. Quatrième de couverture de la collection « L'espèce humaine » de Gallimard. Le texte n'est pas signé, mais il a été très vraisemblablement rédigé par Michel Leiris.

7. «À la suite de nos derniers poètes artistes, artistes et musiciens, la faveur des élites se porte vers l'art des peuples réputés primitifs et sauvages. [...] Ceci provoque dans l'ethnographie d'étranges incursions, accroît une confusion qu'on prétendait réduire. Le Trocadéro rénové pouvait se fonder sur ce contresens, devenir un musée des Beaux-Arts, où les objets se répartiraient sous l'égide de la seule esthétique. Pauvre principe à la vérité, qui n'aboutit qu'à distraire du tableau et au hasard, quelques-uns seulement de ses éléments essentiels. » (GeorgesHenri Rivière, «Le Musée d'ethnographie du Trocadéro », Documents, 1929, n 1, p. 58)

8. Le Musée vivant est le bulletin de l'APAM (Association populaire des amis du musée), créée par Jacques Soustelle, et dont la fondation est annoncée en une de l'hebdomadaire socialiste Vendredi du 26 juin 1936 avec ce mot d'ordre : « Ouvrons les portes de la culture. » Il commence à paraître en février 1937, et propose des extraits de conférences, signale les visites guidées gratuites, donne un « agenda de nos réunions et sorties », etc. 
9. «Un document singulièrement évocateur ", c'est ainsi qu'est décrit Méharées, l'ouvrage de Théodore Monod, lui-même ancien élève de l'Institut d'ethnologie, dans La Terre et la Vie, le bulletin de la Société des amis du Muséum d'histoire naturelle (1937, n 4, p. 62).

10. Lettre à Levasseur du 21 novembre 1835 (citée dans Stendhal 1973 : 354).

11. Voir aussi le préambule de «Vittoria Accoramboni » : «L'auteur inconnu du manuscrit est un personnage circonspect, il ne juge jamais un fait, ne le prépare jamais ; son affaire unique est de raconter avec vérité. Si quelquefois il est pittoresque, à son insu, c'est que vers 1585 , la vanité n'enveloppait point toutes les actions des hommes d'une auréole d'affectation ; [...] personne ne songeait à être aimable par la parole. [...] Ainsi, ô lecteur bénévole! ne cherchez point ici un style piquant, rapide, brillant de fraîches allusions aux façons de sentir à la mode, ne vous attendez point surtout aux émotions entraînantes d'un roman de Georges Sand. » (Ibid., p. 22)

12. Voir, par exemple, cette phrase qu'on trouve en ouverture du Manuel de Mauss: «La sociologie et l'ethnologie descriptive exigent qu'on soit à la fois chartiste, historien, statisticien... et aussi romancier, capable d'évoquer une société tout entière. » (Mauss 1967 [1947] : 8.)

13. Prise à partie dans un compte rendu de L'Homme après la publication de son livre Les Enfants des nuages, Sophie Caratini explique son projet en ces termes : «Peut-être la réticence de l'auteur du compte rendu provient-elle de la forme qui a été donnée à cette anthropologie réflexive : celle d'un récit à plusieurs niveaux de lecture, visant plusieurs publics, français et mauritaniens, néophytes ou savants, et qui, certes, déborde par endroits le champ de l'investigation traditionnel des "scolastiques" de la discipline. Pour ce faire, l'écriture littéraire a pris le pas sur l'écriture scientifique, seul moyen de restituer la trame de l'existence quotidienne dans laquelle les éléments de la démonstration, pour être compréhensibles, devaient être replacés.» («Expérience du terrain et construction du savoir », L’Homme, n 143, juillet- septembre 1997, p. 184). Ainsi, lorsqu'un savant se voit taxé de littérature, n'a-t-il souvent d'autre choix que de combiner deux arguments : la nécessité de toucher un public plus vaste que celui de ses pairs et l'exigence d'une évocation plus fidèle à la réalité de l'enquête que les écrits scientifiques.

14. Paul Rivet, cité par Sébastien Charléty, recteur de l'Université de Paris, qui préface Les Flambeurs d'hommes.

15. Voir cette remarque de Marcel Cohen à propos de Dieu d'eau en 1962 : «quand on sait que Marcel Griaule était affligé d'une véritable mythomanie (contre laquelle il a lui-même lutté dans ses premiers ouvrages), on est obligé de se demander ce qui est de la fabulation ou non. » (Cohen $1962: 91)$

16. En 1951, Griaule avait été pris à partie au cours d'une conférence intitulée « Connaissance de l'homme noir» par un intellectuel africain nommé Taoré (sans doute pour Traoré) en ces termes : «Taoré : Ce que l'on vous reproche, à vous en particulier ethnologues, c'est de dire : "Il y a une civilisation noire." Non, il n'y a pas une civilisation noire.../ Griaule: Si, il y a une civilisation noire./ Taoré : Non, il y en a eu une. » (Griaule 1951 : 163)

\section{RÉSUMÉS}

Publié en 1934, Les Flambeurs d'hommes de Marcel Griaule relate la première «mission Griaule», en 1928, dans la région du Godjam en Ethiopie. Quoique oublié aujourd'hui, l'ouvrage rencontre un succès certain lors de sa parution. Et il mérite qu'on s'y arrête à nouveau en raison de quelques singularités qui le rendent tout à fait unique et sans équivalent dans l'ensemble des récits 
ethnographiques $\mathrm{du} \mathrm{xx}^{\mathrm{e}}$ siècle, à commencer par le fait que Griaule y parle de lui-même à la troisième personne. Cet article s'attache à rendre compte de ces singularités en situant ce curieux récit dans le contexte épistémologique de l'ethnologie de l'entre-deux-guerres. Les Flambeurs d'hommes peut en effet être lu comme un révélateur des contradictions de la discipline à cette époque. Il témoigne en particulier du rapport coupable de l'ethnologie à la littérature, la littérature étant à la fois ce dont il faut se défaire au nom du document et la technique dont on rêve en raison de sa capacité supposée à restituer «l'atmosphère morale» d'une société.

Published in 1934, Marcel Griaule's Les Flambeurs d'hommes [Burners of Men] recounts the first "Griaule mission," undertaken in 1928 in the Godjam region of Ethiopia. Although forgotten today, the book was initially published to considerable success. Indeed, it deserves a critical reappraisal based on several features which make it completely unique and without any equivalent in ethnographic literature of the 20th century, foremost among them Griaule's use of the third person when speaking about himself. To be properly understood, this curious text needs to be situated in the epistemological context of French ethnology of the interwar period. Indeed, Les Flambeurs d'hommes sheds light on the contradictions of the anthropological discipline of that era. In particular, it demonstrates ethnology's ambivalent relationship to literature, as literature is held to be, on the one hand, that which must be discarded on behalf of the objectivity of the document, and, on the other hand, a technique much desired for its alleged ability to convey and restore the "moral atmosphere" of society.

\section{INDEX}

Mots-clés : Griaule, Flambeurs d'hommes, ethnologie, littérature, littérateur, document, évocation, rhétorique

Keywords : Flambeurs d'hommes [Burners of men], anthropology, literature, litterateur/man of letters, rhetoric

\section{AUTEUR}

\section{VINCENT DEBAENE}

Columbia University, Department of French and Romance Philology, vd2169@columbia.edu 